\title{
Measure differential inclusions - between continuous and discrete
}

\author{
Mieczysław Cichoń ${ }^{1}$ and Bianca R Satco ${ }^{2 *}$
}

\author{
"Correspondence: \\ bianca.satco@eed.usv.ro \\ ${ }^{2}$ Faculty of Electrical Engineering \\ and Computer Science, 'Stefan cel \\ Mare' University of Suceava, \\ Universitatii 13, Suceava, Romania \\ Full list of author information is \\ available at the end of the article
}

\begin{abstract}
The paper is devoted to the study of the measure-driven differential inclusions $d x(t) \in G(t, x(t)) d \mu(t), x(0)=x_{0}$ for arbitrary finite Borel measure $\mu$. This type of results allows one to treat in a similar manner differential and difference inclusions, as well as impulsive problems and therefore to study the evolution of hybrid systems with very complex (including Zeno) behavior. Our method is based on viewing the Borel measures as Lebesgue-Stieltjes measures. We thus obtain, under very general assumptions, the existence of regulated or bounded variation solutions of the considered problem and we indicate some advantages of our approach.

MSC: Primary 49N25; secondary 34A60; 93C30; 49J53; 37N35; 34A37
\end{abstract}

Keywords: measure-driven inclusion; Lebesgue-Stieltjes measure; hybrid system

\section{Introduction}

Let us consider the following problem:

$$
\begin{aligned}
& d x(t) \in G(t, x(t)) d \mu(t), \\
& x(0)=x_{0},
\end{aligned}
$$

where $G:[0,1] \times \mathbb{R}^{d} \rightarrow \mathcal{P}\left(\mathbb{R}^{d}\right)$ is a closed convex-valued multifunction and $\mu$ is a positive regular Borel measure. This kind of problems covers some well-known cases like usual differential inclusions (when $\mu$ is the Lebesgue measure), difference inclusions (for discrete measure $\mu$ ) and some impulsive multivalued problems (in the case where the measure $\mu$ can be decomposed as a sum of the Lebesgue measure and a finite sum of Dirac measures-see [1-3]). There are several approaches for the above problems (direct methods or time scale analysis, for instance) but our method, based on Lebesgue-Stieltjes integration, seems to be the most natural.

This kind of results has a long history and is well motivated. A very interesting and well-illustrated course on hybrid inclusions can be found in [4], for instance. Nevertheless, several different approaches, different solutions and many applications for measuredriven equations or inclusions can also be found in the literature. Let us note the paper by Moreau [5]. It is necessary to mention at least some basic papers by Code and Loewen [6], Goebel and Teel [7], Sesekin and Fetisova [8], Silva and Vinter [9], Aubin [1, 2], Ahmed [10], Pereira, Silva and Oliveira [11, 12] and recently by Goncharova and Staritsyn [13], Filippova [14], Lygeros, Quincampoix and Rzeżuchowski [3] and Leine-van de Wouw [15].

\section{Springer}

○2014 Cichon and Satco; licensee Springer. This is an Open Access article distributed under the terms of the Creative Commons Attribution License (http://creativecommons.org/licenses/by/2.0), which permits unrestricted use, distribution, and reproduction in any medium, provided the original work is properly cited. 
Note that in this theory discontinuous functions are usually considered as solutions, which means that we can expect some paradoxes. In particular, different functions can serve as solutions for the same problem in different meanings ( $c f .[16])$. Thus we stress that a crucial aspect of this theory is to fix the definition for the concept of a solution for (1).

We concentrate on two aspects of mentioned papers: we relax the assumptions on $\mu$ and on $G$ by imposing some conditions related to the optimal control theory and we reduce the general problem by working with Lebesgue-Stieltjes integral equations and utilizing their methods and results.

It should be also noted that our results lead in a natural way to some existence theorems for differential and difference inclusions or impulsive problems (for particular measures). Moreover, as claimed in [9], measure-driven differential inclusions provide a convenient framework for formulating optimal control problems for both conventional and impulse controls.

Let us point out that in the context of differential equations this approach has been known for many years. To the best of our knowledge the first equation with a measure as a coefficient was considered by Kronig and Penney in 1931 [17] and as an integral problem by Atkinson [18] in 1964 - in the context of the Riemann-Stieltjes integral. The systematic study for measure-perturbed problems started in the 1970s. It is necessary to mention some basic papers by Sharma [19], Shendge and Joshi [20] or Dhage and Bellale [21]. The method via integral equations for some differential equations (with the Perron-Stieltjes integral) were continued in a systematic way by Schwabik, Tvrdý and Vejvoda [22] in 1979; then some cases have been studied by Wyderka [23, 24], see also [25]. There is still a growing number of papers dealing with measure differential equations ([26] or [27], for instance).

To end the introductory section, we remark that in the considered case there are some difficulties. One of these lies in the lack of natural assumptions implying the existence of suitable integrals (namely, for discontinuous functions the Riemann-Stieltjes integral with respect to bounded variation functions might not be well-defined) and then the question of the chosen definition of the concept of solution. Another problem is how to find (or at least approximate) this solution. For measure-driven differential equations there are some satisfactory answers $(c f$. $[8,27]$ or $[28,29]$ for hybrid systems, for instance). For differential inclusions the situation is more complicated, as will be clarified in what follows. We will also focus on some aspects of this theory, which are related to unification of many multivalued problems (including continuous, discrete, and impulsive systems).

\section{Miscellaneous results from measure theory}

Unlike in the case of usual differential inclusions, the measure theory and the theory of Lebesgue-Stieltjes integrals form the basic tools of our paper. In order to make the paper self-contained we recall all necessary results from these theories.

Let $\mu$ and $v$ be two positive (countably additive) measures on an arbitrary $\sigma$-algebra $\mathcal{A}$ of a space $T$. Recall that:

(i) $v$ is said to be absolutely continuous with respect to $\mu$ if $v(A)=0$ whenever $\mu(A)=0$.

(ii) $v$ and $\mu$ are said to be mutually singular if there exists a measurable set $A$ with $\mu(A)=v(T \backslash A)=0$. 
Recall also of the following notions:

(iii) A set $E \in \mathcal{A}$ is an atom of the measure $\mu$ if $\mu(E)>0$ and each measurable subset $F$ of $E$ has either $\mu(F)=0$ or $\mu(F)=\mu(E)$.

(iv) A measure is called nonatomic (or diffuse, in probability theory) if it has no atoms.

(v) We shall say that a measure is purely atomic if every measurable set of positive measure contains an atom.

Two atoms $E_{1}$ and $E_{2}$ are said to be non-equivalent if $d\left(E_{1}, E_{2}\right)=\mu\left(E_{1} \Delta E_{2}\right)>0$. In this case $\mu\left(E_{1} \cap E_{2}\right)=0$. Any $\sigma$-finite measure has at most a countable number of pairwise non-equivalent atoms (e.g. [30, p.55]).

It is well known (the Lebesgue decomposition theorem) that if the measures $\mu$ and $v$ are $\sigma$-finite, then $v$ can be written, in a unique way, as a sum of a measure $v_{a c}$ which is absolutely continuous with respect to $\mu$ and a measure $v_{s}$ singular with respect to $\mu$ :

$$
v=v_{a c}+v_{s}
$$

In particular, if $v$ is nonatomic (as is the case of the Lebesgue measure) then $v_{a c}$ is also nonatomic. Moreover, the singular part can be (uniquely again) decomposed in a sum of a purely atomic and a nonatomic measure (the Cantor part) (see [31], for instance):

$$
v_{s}=v_{n s}+v_{p a} .
$$

Thus the measure $v$ can be decomposed as a sum of three mutually singular measures: the Lebesgue part, the Cantor part and the singular (or jump) part, $c f$. [16, 32]

$$
v=v_{a c}+v_{n s}+v_{s}
$$

We are interested in using Borel measures to solve the problem announced in the Introduction. The classical Riesz Representation Theorem characterizes the finite regular Borel measures on a compact metrizable space as linear continuous functionals on the space of real continuous functions. This characterization is used by most of the authors studying measure-driven equations, e.g. $[9,23]$.

We prefer here another approach, based on Lebesgue-Stieltjes integration. First of all, recall ([33, p.438] or [34, p.126]) that any finite Borel measure on a Polish space (in particular on the unit interval of the real line) is regular. It was shown that every finite Borel measure on the real line agrees with some Lebesgue-Stieltjes measure restricted to the class of Borel sets. More precisely we have:

Theorem 1 [34, Theorem 3.21] Let $\mu$ be a Borel measure on $\mathbb{R}$ with $\mu(B)<\infty$ for every bounded Borel set $B$. Then there exists a nondecreasing, right-continuous function $F: \mathbb{R} \rightarrow$ $\mathbb{R}$ such that $\mu(B)=\mu_{F}(B)$ for any Borel set $B$.

Here $\mu_{F}$ denotes the Lebesgue-Stieltjes measure with distribution function $F$. Moreover, it was shown (see [35, pp.236-238]) that:

- The measure is nonatomic if and only if $F$ is continuous.

- The measure is absolutely continuous with respect to the Lebesgue measure if and only if $F$ is absolutely continuous. 
- The measure is nonatomic and singular with respect to the Lebesgue measure if and only if $F$ is a singular function.

Recall that a singular function is a function which is continuous and nondecreasing on a real interval and has the derivative 0 except for a set of zero Lebesgue measure. There exist non-constant singular functions on the unit interval (e.g. [34, p.282]), examples of such functions being in general constructed using the Cantor set.

Concerning the atoms of Lebesgue-Stieltjes measures, it can be seen in [34, p.132] or [36, $211 \mathrm{X}]$ that each atom $E$ contains a singleton $a \in E$ for which $\mu_{F}(E)=\mu_{F}(\{a\})$. Comparing to definition (v) above, this explains why in some references (such as [33]) a measure $\mu$ is called purely atomic if there exists a countable set $A$ such that the outer measure of each singleton $\{a\}, a \in A$ is strictly positive and the outer measure of the complementary of $A$ is 0 . Obviously, if each singleton is measurable, then the measure takes the place of the outer measure in the previous sentence.

Then any Lebesgue-Stieltjes measure $\mu_{F}$ (associated with $F$ ) may be split into a sum of three measures: discrete, absolutely continuous and singular ones.

As we will prove our results via Lebesgue-Stieltjes measures, it is worthwhile to recall some basic properties of Lebesgue-Stieltjes integrals; they are consequences of Proposition 2.3.16 in [27], where the functions are real-valued and the integral under consideration is the Perron-Stieltjes one (which is even more general than the Lebesgue-Stieltjes integral). For generalized integral equations, investigated also independently of our motivations, the class of regulated functions plays a major role. Solutions for integral problems involving Lebesgue-Stieltjes or Perron-Stieltjes integrals should be investigated outside the space of continuous functions or the spaces of functions with bounded variation. It was clarified in [27] that the space of regulated functions is the best choice for the space of solutions.

Since the notion of regulated functions has sometimes different meanings, we need to describe this class of functions.

Definition 2 A function $F:[0,1] \rightarrow \mathbb{R}$ is said to be regulated if there exist the limits $F(t+)$ and $F(s-)$ for all points $t \in[0,1)$ and $s \in(0,1]$.

It is well known that the set of discontinuities for a regulated function is at most countable, but such a function need not be of bounded variation. Moreover, a function $F:[0,1] \rightarrow \mathbb{R}$ is regulated if and only if it is a uniform limit of a sequence of finite step functions. The following property of the indefinite Kurzweil-Stieltjes integral implies that solutions of measure differential equations are regulated functions. This can explain when our solutions are either of bounded variation or regulated.

Proposition 3 [27, Proposition 2.3.16] Let $F:[0,1] \rightarrow \mathbb{R}$ and $g:[0,1] \rightarrow \mathbb{R}^{d}$ be such that the Lebesgue-Stieltjes $\int_{0}^{1} g(s) d F(s)$ exists. Then:

(i) If $F$ is regulated, then so is the primitive $h:[0,1] \rightarrow \mathbb{R}^{d}, h(t)=\int_{0}^{t} g(s) d F(s)$ and for every $t \in[0,1]$,

$$
h\left(t^{+}\right)-h(t)=g(t)\left[F\left(t^{+}\right)-F(t)\right] \text { and } h(t)-h\left(t^{-}\right)=g(t)\left[F(t)-F\left(t^{-}\right)\right] .
$$

(ii) If $F$ is of bounded variation and $g$ is bounded, then $h$ is also of bounded variation. 
By $\chi_{A}$ we will denote the characteristic function of the set $A \subset \mathbb{R}$. We also need some preliminary facts from set-valued analysis.

The family of all nonempty closed (respectively, nonempty closed convex) subsets of $\mathbb{R}^{d}$ will be denoted by $\mathcal{P}_{c}\left(\mathbb{R}^{d}\right)$ (respectively, $\mathcal{P}_{c c}\left(\mathbb{R}^{d}\right)$ ). By the distance between a point $x \in \mathbb{R}^{d}$ and a set $A \subset \mathbb{R}^{d}$ we mean

$$
d(x, A)=\inf \{\|x-a\|: a \in A\} .
$$

Let us recall that a multifunction $\Gamma:[0,1] \rightarrow \mathcal{P}_{c}\left(\mathbb{R}^{d}\right)$ is said to be (Hausdorff-) upper semicontinuous, shortly usc, at a point $t_{0} \in[0,1]$ if for every $\varepsilon>0$ there exists $\delta_{\varepsilon}>0$ such that the excess of $\Gamma(t)$ over $\Gamma\left(t_{0}\right)$ (in the sense of Hausdorff) is less than $\varepsilon$ whenever $\left|t-t_{0}\right|<\delta_{\varepsilon}$. Otherwise stated,

$$
\Gamma(t) \subset \Gamma\left(t_{0}\right)+\varepsilon B
$$

where $B$ is the unit ball of $\mathbb{R}^{d}$. In the obvious way we call a multifunction usc when it is usc at each point $t_{0} \in[0,1]$.

\section{Main results}

The solutions of usual differential problems are at least continuous, but if we look at, for example, a big part of the class of hybrid systems, this is not available. In our problems, by considering inclusions driven by general Borel measures, it cannot be expected to obtain continuous solutions. This is the reason for which in the following definition the using of the left limit:

$$
x\left(t^{-}\right)=\lim _{\tau \rightarrow t, \tau<t} x(\tau)
$$

is crucial. Indeed, as pointed out in an example in [37], taking the integral on a closed, resp. open interval (which is equivalent to integrating on a closed interval the left limit of the function) leads to completely different solutions.

Definition 4 A solution of the problem (1) is a function $x:[0,1] \rightarrow \mathbb{R}^{d}$ for which there exists a $\mu$-integrable function $g:[0,1] \rightarrow \mathbb{R}^{d}$ such that

$$
x(t)=x_{0}+\int_{0}^{t} g(s) d \mu(s), \quad \forall t \in[0,1]
$$

and

$$
g(t) \in G\left(t, x\left(t^{-}\right)\right) \quad \mu \text {-a.e. }
$$

Let us note that for discontinuous solutions there is always a problem how to define a solution in the points of discontinuity. A very interesting survey on the topic can be found in [16]. We need to remark that distinct definitions of solutions can lead to distinct solutions [38]! The existence and uniqueness of solutions of considered problems depend on the conditions for $\mu$ and $g$. Due to the existence of atoms for the measure $\mu$ there is a question about the uniqueness of solutions for a (possibly) discontinuous function $g$. The 
explicit scheme $\Delta x(t)=x(t)-x\left(t^{-}\right)=g\left(x\left(t^{-}\right)\right)$is a natural choice for physical systems and we will follow this idea. This allows us to fill the gap in this theory. If we compare our result with some earlier ones we need to recall that for purely atomic measure $\mu$ the above condition of integrability means that the series $\sum_{k} g\left(t_{k}\right) \mu\left\{t_{k}\right\}$ is finite (where $t_{k}$ is a set of atoms for $\mu)-c f$. $[8,16]$, for instance.

Let us present some auxiliary result:

Lemma 5 Let $\Gamma: \mathbb{R}^{d} \rightarrow \mathcal{P}_{c c}\left(\mathbb{R}^{d}\right)$ be an usc multifunction and $\left(x_{n}\right)_{n}$ be a sequence that converges to $x \in \mathbb{R}^{d}$. Suppose that there exists a constant $M>0$ such that $d\left(0, \Gamma\left(x_{n}\right)\right) \leq M$ for every $n \in \mathbb{N}$. Then

$$
d(0, \Gamma(x)) \leq M
$$

Proof By the upper semicontinuity of $\Gamma$ it follows that $d(0, \Gamma(\cdot))$ is lower semicontinuous (see [39, Lemma 9.3.1]). Therefore

$$
d(0, \Gamma(x)) \leq \liminf _{n \rightarrow \infty} d\left(0, \Gamma\left(x_{n}\right)\right) \leq M .
$$

We are ready to present our first result for measure-driven differential inclusions (1) for a general class of finite Borel measures. Let us note that the presented theorem is intended to unify and to extend the earlier ones. We not only formulate an existence result, but we also include a method how to find this solution as a limit of some approximations. We refer the reader to [16] for the discussion, some motivations and examples for measure-driven problems.

Theorem 6 Let $\mu$ be a finite Borel measure on $[0,1]$ and let $G:[0,1] \times \mathbb{R}^{d} \rightarrow \mathcal{P}_{c c}\left(\mathbb{R}^{d}\right)$ satisfy the following hypotheses:

(1) $G(\cdot, \cdot)$ is product Borel measurable,

(2) $G(t, \cdot)$ is usc for every $t \in[0,1]$,

(3) there exists a $\mu$-integrable function $M:[0,1] \rightarrow \mathbb{R}_{+}$such that

$$
d(0, G(t, y)) \leq M(t), \quad \forall t \in[0,1], y \in \mathbb{R}^{d} .
$$

Then there exists at least one solution for the measure-driven differential problem (1).

Proof Our proof is based on an iteration procedure. More precisely, we construct a sequence of approximate solutions (being regulated functions) which is shown to have a convergent subsequence due to some compactness properties.

So, let $x^{0}(t)=x_{0}$ for $t \in[0,1]$. Suppose then that we have already constructed a regulated (bounded variation (BV)) function $x^{n}$ on $[0,1]$ and choose $x^{n+1}$ by following a scheme that is described in the sequel.

By our hypotheses on $G$ we ensure that the function $G$ is superpositionally Borel measurable [40]. Since $x^{n}$ is regulated (BV), there exists $x^{n}\left(t^{-}\right)$at every point $t$. It can be obtained as $x^{n}\left(t^{-}\right)=\lim _{m \rightarrow \infty} x^{n}\left(t-\tau_{m}\right)$, where $\left(\tau_{m}\right)_{m}$ is a sequence of positive numbers converging to 0 (such that $\left.t-\tau_{m} \in[0,1]\right)$. Therefore the function $t \mapsto x^{n}\left(t^{-}\right)$is measurable as a pointwise limit of a sequence of measurable functions and then the multifunction $t \mapsto G\left(t, x^{n}\left(t^{-}\right)\right)$is Borel measurable too. 
By Theorem 12.1 in [41] ( $c f$. also Chapter III in [42]) it follows that $t \mapsto d\left(0, G\left(t, x^{n}\left(t^{-}\right)\right)\right)$is Borel measurable. Moreover, by hypothesis (3) and Lemma 5, it is bounded by $M(t)$. Since the values of $G$ are closed and convex we are able to find a Borel measurable selection $g^{n}(\cdot)$ of $G\left(\cdot, x^{n}\left(\cdot \cdot^{-}\right)\right)$such that

$$
\left\|g^{n}(t)\right\|=d\left(0, G\left(t, x^{n}\left(t^{-}\right)\right)\right) \quad \text { for every } t \in[0,1]
$$

(in our finite-dimensional case, it is unique). Define now

$$
x^{n+1}(t)=x_{0}+\int_{0}^{t} g^{n}(s) d \mu(s), \quad \forall t \in[0,1] .
$$

As it was presented in the preliminary part of the paper (Theorem 1 ), the measure $\mu$ is, in fact, a Lebesgue-Stieltjes measure with respect to a BV, right-continuous function $F$. Thus the previous integral should be understood in the sense of $\int_{0}^{t} g^{n}(s) d \mu_{F}(s)$ i.e. as a LebesgueStieltjes integral. This integral is well defined since the selection $g^{n}$ is Borel measurable, $F$ is of bounded variation and, as said before, bounded by $M(t)$. Moreover, by Proposition 3, $x^{n+1}$ is a regulated function.

The function $F$ is of bounded variation and right-continuous, therefore it has at most countable points of left discontinuity. Let $A=\left\{t_{k}: k \in \mathbb{N}\right\}$ be the set of its discontinuity points.

As the sequence $\left(g^{n}\right)_{n}$ is pointwise bounded, we can extract, by a diagonal procedure, a subsequence (not re-labeled) for which

$$
g^{n}\left(t_{k}\right) \rightarrow \widetilde{g}\left(t_{k}\right), \quad \forall k \in \mathbb{N}
$$

As a pointwise limit of measurable functions $\widetilde{g}$ is measurable on $A$. By (3), using the Lebesgue dominated convergence result Theorem 5.3.3 in [43], we obtain for every $t \in$ $[0,1]$

$$
\int_{[0, t] \cap A} g^{n}(s) d \mu_{F}(s) \rightarrow \int_{[0, t] \cap A} \tilde{g}(s) d \mu_{F}(s) .
$$

At the same time, the sequence $\left(g^{n} \chi_{[0,1] \backslash A}\right)_{n}$ is uniformly integrable in $L^{1}\left([0,1] \backslash A, \mu_{F}\right)$ and bounded. Whence it is relatively weakly compact in the space $L^{1}\left([0,1] \backslash A, \mu_{F}\right)(c f .[44])$. It follows that we can extract a subsequence (denoted in the same way, for the sake of convenience) which converges in the weak topology of $L^{1}\left([0,1] \backslash A, \mu_{F}\right)$ to some function $h \in L^{1}\left([0,1] \backslash A, \mu_{F}\right)$. In particular,

$$
\int_{[0, t] \backslash A} g^{n}(s) d \mu_{F}(s) \rightarrow \int_{[0, t] \backslash A} h(s) d \mu_{F}(s), \quad \forall t \in[0,1] .
$$

Denoting now by

$$
g=\tilde{g} \chi_{A}+h \chi[0,1] \backslash A,
$$

we get

$$
\int_{0}^{t} g^{n}(s) d \mu_{F}(s) \rightarrow \int_{0}^{t} g(s) d \mu_{F}(s), \quad \forall t \in[0,1] .
$$


Then the function defined by

$$
x(t)=x_{0}+\int_{0}^{t} g(s) d \mu_{F}(s), \quad \forall t \in[0,1],
$$

has the property that the subsequence of $\left(x^{n}\right)_{n}$ is pointwise convergent to $x$.

We assert that the function $x$ obtained by this procedure is a solution to our problem. For this purpose, it suffices to prove that

$$
g(s) \in G\left(s, x\left(s^{-}\right)\right) \quad \mu_{F} \text {-a.e. }
$$

The first step in this direction is to show that

$$
\lim _{n \rightarrow \infty} x^{n}\left(t^{-}\right)=x\left(t^{-}\right) \quad \mu_{F} \text {-a.e. }
$$

For any $t \in[0,1] \backslash A$, by Proposition 3,

$$
x^{n}(t)-x^{n}\left(t^{-}\right)=g^{n-1}(t)\left[F(t)-F\left(t^{-}\right)\right]=0
$$

and

$$
x(t)-x\left(t^{-}\right)=g(t)\left[F(t)-F\left(t^{-}\right)\right]=0,
$$

therefore

$$
x^{n}\left(t^{-}\right)=x^{n}(t) \rightarrow x(t)=x\left(t^{-}\right) .
$$

Let now $t$ be an element of $A$. In this case,

$$
x^{n}(t)-x^{n}\left(t^{-}\right)=g^{n-1}(t)\left[F(t)-F\left(t^{-}\right)\right]
$$

and

$$
x(t)-x\left(t^{-}\right)=g(t)\left[F(t)-F\left(t^{-}\right)\right],
$$

whence

$$
x^{n}\left(t^{-}\right)-x\left(t^{-}\right)=x^{n}(t)-x(t)+\left[g(t)-g^{n-1}(t)\right]\left[F(t)-F\left(t^{-}\right)\right]
$$

and it can be seen that it tends to 0 since $x^{n}(t) \rightarrow x(t), g^{n-1}(t) \rightarrow g(t)$ and $\left\|F(t)-F\left(t^{-}\right)\right\| \leq$ $\|F\|_{\mathrm{BV}}<\infty$.

At the next step, by the upper semicontinuity assumption on $G(t, \cdot)$, it follows that for every $\varepsilon>0$ and $s \in[0,1]$ there exists $n_{\varepsilon, s} \in \mathbb{N}$ such that

$$
G\left(s, x^{n}\left(s^{-}\right)\right) \subset G\left(s, x\left(s^{-}\right)\right)+\varepsilon B, \quad \forall n \geq n_{\varepsilon, s} .
$$


Finally, since $\left(g^{n}\right)_{n}$ is weakly convergent in $L^{1}\left([0,1] \backslash A, \mu_{F}\right)$, following Theorem 2.1 in [44], there exists a sequence $\left(\bar{g}^{n}\right)_{n}$ of convex combinations of $\left\{g^{m}: m \geq n\right\}$ which is pointwise $\mu_{F}$-a.e. convergent on $[0,1] \backslash A$. By a classical method (using the Lebesgue dominated convergence theorem) it can be shown that this limit is equal $\mu_{F}$-a.e. to $h$. As $\bar{g}^{n}(s) \in G\left(s, x\left(s^{-}\right)\right)+\varepsilon B$ for all $n \geq n_{\varepsilon, s}$, by passing to the limit, we get $h(s) \in G\left(s, x\left(s^{-}\right)\right)$, $\mu_{F}$-a.e. on $[0,1] \backslash A$. From this relation and taking into account (3) we obtain

$$
g(s) \in G\left(s, x\left(s^{-}\right)\right) \quad \mu_{F} \text {-a.e. }
$$

and so, $x$ is a solution.

Remark 7 The solutions are, following Proposition 3, regulated. In the particular case where $M$ is bounded, all approximate solutions $x^{n}$ and our solution too are, in fact, of bounded variation.

Moreover, when $\mu$ is absolutely continuous with respect to the Lebesgue measure we obtain a result related to that in [45, Theorem 4, p.101]. Our theorem provides the existence of slow solutions for the considered problem in the sense of [45, Definition 1, p.97].

Under a different boundedness assumption, very natural and well known in Lebesgue integration, we get another existence result.

Theorem 8 Let the finite Borel measure $\mu$ on $[0,1]$ and $G:[0,1] \times \mathbb{R}^{d} \rightarrow \mathcal{P}_{c c}\left(\mathbb{R}^{d}\right)$ satisfy hypotheses (1), (2) in Theorem 6 and

$\left(3^{\prime}\right)$ there exist a positive function $M \in L^{1}([0,1], \mu)$ and a constant $N>0$ such that $G(t, y) \subset$ $[M(t)+N\|y\|] B$ for all $t \in[0,1]$ and $y \in \mathbb{R}^{d}$.

Then there exists at least one solution for the measure-driven differential problem (1) on some interval $[0, T] \subset[0,1]$.

Proof First, as the map $t \mapsto \int_{0}^{t} M(s) d \mu(s)$ is regulated, it is bounded on [0,1] by some $M>0$. Also, denoting by $K(t)=\int_{0}^{t} d \mu(s)$, we get a nondecreasing function. Since 0 is not a point of discontinuity, one can choose $T \in[0,1]$ such that $N K(T)<1$.

In the sequel, following the same iterative method as in the proof of Theorem 6 , we choose $x^{0}(t)=x_{0}$ for $t \in[0,1]$ and then we pick $x^{1}$ as follows. The function $G$ is superpositionally Borel measurable, thus we are able to choose a Borel measurable selection $g^{0}(\cdot)$ of $G\left(\cdot, x^{0}\left(\cdot \cdot^{-}\right)\right)$(see [42, p.59]). Define

$$
x^{1}(t)=x_{0}+\int_{0}^{t} g^{0}(s) d \mu(s), \quad \forall t \in[0,1]
$$

By hypotheses,

$$
\left\|x^{1}(t)\right\| \leq\left\|x_{0}\right\|+\int_{0}^{t} M(s)+N\left\|x_{0}\right\| d \mu(s)
$$

and so for all $t \in[0,1]$,

$$
\left\|x^{1}(t)\right\| \leq\left\|x_{0}\right\|+M+N K(t)\left\|x_{0}\right\|=M+\left\|x_{0}\right\|(1+N K(t)) \leq M+\frac{\left\|x_{0}\right\|}{1-N K(t)} .
$$


Similarly, because $K$ is nondecreasing, one can estimate

$$
\left\|x^{2}(t)\right\| \leq M(1+N K(t))+\left\|x_{0}\right\|\left(1+N K(t)+N^{2} K^{2}(t)\right), \quad \forall t \in[0,1] .
$$

We are able to prove by the method of mathematical induction that for all $t \in[0, T]$

$$
\left\|x^{n}(t)\right\| \leq M\left(1+\cdots+N^{n-1} K^{n-1}(t)\right)+\left\|x_{0}\right\|\left(1+N K(t)+\cdots+N^{n} K^{n}(t)\right) \leq \frac{M+\left\|x_{0}\right\|}{1-N K(t)} .
$$

For $n=1$ it is already verified. Now, constructed $x^{n}$, we proceed as before and choose a $\mu$-integrable selection $g^{n}$ of $G\left(\cdot, x^{n}\left(\cdot \cdot^{-}\right)\right)$. Define

$$
x^{n+1}(t)=x_{0}+\int_{0}^{t} g^{n}(s) d \mu(s), \quad \forall t \in[0,1] .
$$

So,

$$
\begin{aligned}
\left\|x^{n+1}(t)\right\| \leq & \left\|x_{0}\right\|+\int_{0}^{t} M(s)+N\left\|x^{n}(s)\right\| d \mu(s) \\
\leq & \left\|x_{0}\right\|+M+N \int_{0}^{t} M\left(1+\cdots+N^{n-1} K^{n-1}(s)\right) \\
& +\left\|x_{0}\right\|\left(1+N K(s)+\cdots+N^{n} K^{n}(s)\right) d \mu(s) \\
= & \left\|x_{0}\right\|+M+N\left[M\left(1+\cdots+N^{n-1} K^{n-1}(t)\right)\right. \\
& \left.+\left\|x_{0}\right\|\left(1+N K(t)+\cdots+N^{n} K^{n}(t)\right)\right] K(t) \\
= & M\left(1+\cdots+N^{n} K^{n}(t)\right)+\left\|x_{0}\right\|\left(1+N K(t)+\cdots+N^{n+1} K^{n+1}(t)\right) \\
\leq & \frac{M+\left\|x_{0}\right\|}{1-N K(t)}, \quad \forall t \in[0, T] .
\end{aligned}
$$

The rest of the proof goes as before, and thus the existence of solutions in the considered sense is achieved.

By applying an appropriate version of Gronwall's lemma we will be able to prove that, in fact, under the assumptions of the previous theorem, one can find global solutions.

Let us recall the following Gronwall-type result (where $D_{\mu}$ denotes the set of all atoms of $\mu$ ):

Lemma 9 [46, Lemma 8.5] Let $\mu=\mu_{c}+\mu_{s}$ be a finite Borel measure on [0,1] with $\mu(\{0\})=0, u:[0,1] \rightarrow \mathbb{R}$ be regulated and $\mu$-integrable, $N>0$ and $M:[0,1] \rightarrow \mathbb{R}_{+}$be nondecreasing and $\mu$-integrable. Suppose that

$$
u(t) \leq M(t)+N \cdot \int_{0}^{t} u(s) d \mu(s), \quad t \in[0,1] .
$$

Then

$$
u(t) \leq M(t)+N \cdot \varphi_{0}(t) \int_{0}^{t} \varphi_{0}^{-1}(s) M(s-) d \mu(s)
$$


where

$$
\varphi_{0}(t)=\exp N \mu_{c}(t) \prod_{\tau \in D_{\mu} \cap[0, t]}(1+N \cdot \Delta \mu(\tau)) .
$$

Remark 10 As $M$ is a $\mu$-integrable function and $s \mapsto \exp (\mu([s, t]))$ is measurable and bounded, their product $s \mapsto M(s) \exp (\mu([s, t]))$ is $\mu$-integrable on $[0, t]$. Denote by

$$
\exp [s, t]=\varphi_{0}(t) \varphi_{0}^{-1}(s)
$$

Then under the conditions of the above lemma (cf. [47, Lemma 2.1]):

$$
u(t) \leq M(t)+N \cdot \int_{0}^{t} M(s) \exp (\mu([s, t])) d \mu(s), \quad t \in[0,1] .
$$

Recall that $\varphi_{0}(t) \leq \exp \operatorname{Var}_{[0, t]} \mu$ and the latest is increasing and of bounded variation.

Theorem 11 Let the finite Borel measure $\mu$ on $[0,1]$ and $G:[0,1] \times \mathbb{R}^{d} \rightarrow \mathcal{P}_{c c}\left(\mathbb{R}^{d}\right)$ satisfy hypotheses in Theorem 8. Then there exists at least one global solution for the measuredriven differential problem (1).

Proof Step I. We firstly prove that we have an a priori estimation for all solutions. Indeed, if $x$ would be an arbitrary solution, then

$$
\begin{aligned}
\left\|x\left(t^{-}\right)\right\| & =\lim _{\tau \rightarrow t, \tau<t}\|x(\tau)\| \\
& \leq\left\|x_{0}\right\|+\lim _{\tau \rightarrow t, \tau<t} \int_{0}^{\tau}\|g(s)\| d \mu(s) \\
& \leq\left\|x_{0}\right\|+\lim _{\tau \rightarrow t, \tau<t} \int_{0}^{\tau}\left[M(s)+N\left\|x\left(s^{-}\right)\right\|\right] d \mu(s) \\
& \leq\left\|x_{0}\right\|+\int_{0}^{t} M(s) d \mu(s)+N \int_{0}^{t}\left\|x\left(s^{-}\right)\right\| d \mu(s),
\end{aligned}
$$

whence, by Lemma 9 ,

$$
\begin{aligned}
& \left\|x\left(t^{-}\right)\right\| \\
& \quad \leq\left\|x_{0}\right\|+\int_{0}^{t} M(s) d \mu(s)+N \int_{0}^{t}\left[\left\|x_{0}\right\|+\int_{0}^{s} M(u) d \mu(u)\right] \exp (\mu([s, t])) d \mu(s)
\end{aligned}
$$

for every $t \in[0,1]$.

Denoting

$$
\tilde{N}(t)=\left\|x_{0}\right\|+\int_{0}^{t} M(s) d \mu(s)+N \int_{0}^{t}\left[\left\|x_{0}\right\|+\int_{0}^{s} M(u) d \mu(u)\right] \exp (\mu([s, t])) d \mu(s),
$$

we obtain a regulated, therefore bounded function so that all solutions $x$ satisfy

$$
\left\|x\left(t^{-}\right)\right\| \leq \tilde{N}(t) \leq K, \quad \forall t \in[0,1] .
$$


Step II. At this step, by a truncation procedure (as was done in the classical continuous case in [48, Theorem 5.2]), define

$$
\widetilde{G}(t, y)= \begin{cases}G(t, y) & \text { if }\|y\| \leq K, \\ G\left(t, \frac{K y}{\|y\|}\right) & \text { if }\|y\|>K .\end{cases}
$$

The obtained multifunction satisfies the following conditions:

(1) $\widetilde{G}(\cdot, \cdot)$ is product Borel measurable,

(2) $\widetilde{G}(t, \cdot)$ is usc for every $t \in[0,1]$,

(3) there exists a $\mu$-integrable function $M(t)+N K$ such that

$$
\widetilde{G}(t, y) \subset(M(t)+N K) B, \quad \forall t \in[0,1], y \in \mathbb{R}^{d} .
$$

Then, by Theorem 6, there exists at least one solution for the measure-driven differential problem

$$
\begin{aligned}
& d x(t) \in \widetilde{G}(t, x(t)) d \mu(t), \\
& x(0)=x_{0} .
\end{aligned}
$$

Step III. Since on both branches $\widetilde{G}(t, y) \subset[M(t)+N\|y\|] B$ for all $t \in[0,1]$ and $y \in \mathbb{R}^{d}$ we can show, by using Gronwall's Lemma like at Step I, that any solution obtained at Step II satisfies the inequality $\|x(t)\| \leq K$ and so, it is a solution of the initial value problem (1).

Remark 12 (i) As it can be seen in our results, the usual assumptions in set-valued analysis are used in a natural way and conduct to existence results for a finite Borel measuredriven differential inclusion. Of course, in the proof, the measure-theory results available for complete measures, such as equivalent definitions of measurability of multifunctions (cf. [42, Chapter III] or [41]), must be avoided.

(ii) Let us now notice that in the main theorems we replaced the condition that $G$ is (locally) Lipschitz (which was used in most of the previously obtained existence results for measure-driven inclusions) by its upper semicontinuity. Moreover, comparing to [9] (which also considers upper semicontinuous multifunctions), we imposed only the upper semicontinuity with respect to the second argument, along with a jointly measurability condition. Since such a kind of results has many applications in the control theory, it seems to be an important improvement. It is very natural to require this kind of assumptions instead of stronger ones. For differential inclusions this is sufficient for proving upper semicontinuity of the solution mapping and then to find an optimal control (cf. [49] or [16, Chapter 6], for instance).

Example 13 The bouncing ball system models an elastic ball bouncing on a (not-necessarily flat) surface. A set-valued bouncing ball i.e. when time dynamics can be set-valued are typically used for deriving bounds on the solution of nonlinear single-valued hybrid systems in a small neighborhood of a Zeno equilibrium point (that is, a point towards which the infinite number of discrete transitions occurring in finite time accumulates, $c f$. [50]). The vertical motion of the ball can be captured by an impulse differential inclusion. 
This system is described by a measure-driven inclusion (which is a reformulated problem from [50])

$$
d x \in F(x) d x+G(x) d \mu_{d}(x)
$$

The considered in this model dynamical system is a sum of a slow-time velocity belonging to a set $F(x)$ and a fast-time contribution coming from another set $G(x) d \mu_{s}$, where $\mu_{s}$ is a vector-valued discrete measure.

In a classical set-valued bouncing ball case we have Lipschitz assumptions on the righthand side - $c f$. [50] or [3]. Namely, we have (cf. [50]) $F(x)=\left\{\left[x_{2}, a\right]: a \in\left[a_{\min }, a_{\max }\right]\right\}, G(x)=$ $\left[0,-e x_{2}\right]$ (with $e<1$ ). The measure $\mu_{d}$ is defined by its distribution

$$
d(t)= \begin{cases}0 & \text { when } 0 \leq t \leq t_{1} \\ -\sum_{j=1}^{i} x_{2}\left(t_{j}-\right) & \text { when } t_{i} \leq t \leq t_{i+1}\end{cases}
$$

Our existence results allow to assert that the problem possess solutions in the more general case where both multifunctions can be time-dependent and only upper semicontinuous.

In a series of papers of Silva and his collaborators (e.g. [9]) another definition for the solution was considered (going back to [51]). The main idea is to use a reparametrization method for $\mu$ and in this way to transform the measure-driven differential inclusions into usual differential inclusions.

In earlier works a kind of limit solutions $x$ was considered (i.e. $x$ is a limit of approximated solutions $x_{n}$ for problems driven by measures $\mu_{n}$ tending, in some sense, to $\mu$ $c f$. [52]). More precisely, the measure $\mu$ was approximated by measures $\mu_{n}$ which are absolutely continuous with respect to the Lebesgue measure and the limit was shown to be independent on the choice of this sequence $\left(\mu_{n}\right)_{n}$, see [46]. A similar concept (approximable solutions) for particular measures can be found in $[8,16]$.

It is interesting that these two definitions are equivalent and can be described as follows.

Definition 14 A function $x:[0,1] \rightarrow \mathbb{R}^{d}$ is called a (robust) solution for the problem (1) if

$$
x(t)=x_{0}+\int_{0}^{t} g(s) d \mu(s), \quad \forall t \in[0,1]
$$

for some $\mu$-integrable function $g$ such that

$$
g(t) \in \widetilde{G}\left(t, x\left(t^{-}\right) ; \mu(\{t\})\right) \quad \mu \text {-a.e., }
$$

where the multifunction $\widetilde{G}$ is defined on $[0,1] \times \mathbb{R}^{d} \times[0, \infty)$ as follows: if $\alpha>0$, then

$$
\widetilde{G}(t, v, \alpha)=\left\{\frac{y(\alpha)-v}{\alpha}: y \in A C^{1}([0, \alpha]), \dot{y}(\sigma) \in G(t, y(\sigma)) \text { a.e., } y(0)=v\right\}
$$

and if $\alpha=0$, then

$$
\widetilde{G}(t, v, \alpha)=G(t, v) .
$$


In some particular cases (as, for example, the problem studied in [3]) our Definition 4 of a solution is equivalent to that of a robust solution. Obviously, for a general Borel measure $\mu$ the two concepts are distinct.

Remark 15 Under the assumptions that $G(\cdot, \cdot)$ has closed graph and the values of $G$ are contained in some ball, Theorem 5.1 in [9] states that the problem (1) has robust solutions. Besides, the solution set is continuous with respect to the data, in the sense that when a sequence of measures $\left(\mu_{i}\right)$ tends to $\mu$ in the weak* topology (cf. [10]), for any sequence $\left(x_{i}\right)$ of robust solutions corresponding to $\mu_{i}$ there exists a robust solution $x$ corresponding to $\mu$ with the property that on a subsequence

$$
x_{i} \rightarrow x\left(\text { weakly }^{*}\right) \text { and } x_{i}(t) \rightarrow x(t) \text { except on the atoms of } \mu \text {. }
$$

Our concept of solution does not offer this closure property (as stated in [9, p.731]), which is essential in some cases (see [51] or [9]), but we consider more general problems and in many situations, for non-robust systems (see $[16,46,53]$ ) solutions as in our Definition 4 are also good enough.

However, a closure property is available: Theorem 6.3 in [25] shows that if $g_{k}(\cdot, x(\cdot)) \rightarrow$ $g(\cdot, x(\cdot))$ w.r.t. the Alexiewicz norm (i.e. the supremum norm of the primitive) for each $x$ and satisfy a Lipschitz condition, then the solutions of the problems

$$
x(t)=x_{0}+\int_{0}^{t} g_{k}\left(s, x\left(s^{-}\right)\right) d \mu(s)
$$

pointwise converge to a solution of the problem

$$
x(t)=x_{0}+\int_{0}^{t} g\left(s, x\left(s^{-}\right)\right) d \mu(s) .
$$

Recall that when considering discontinuous solutions, a fixed measure-driven problem can have solution in one sense and not to have solutions in another sense. Furthemore, different functions can be simultaneously solutions for the same problem-considered in different senses. So, obviously, separate solutions require separate existence theorems.

Until now, we described the problem with an arbitrary Borel measure. One of the possible extensions is to consider for each of the three measures in which our measure can be decomposed $\mu=\mu_{a c}+\mu_{n s}+\mu_{p a}$ (see Section 2) a different set of assumptions. In our main theorem we impose $G d \mu=G_{1} d \mu_{a c}+G_{2} d \mu_{n s}+G_{3} d \mu_{p a}$ with $G=G_{1}=G_{2}=G_{3}$, i.e. the assumptions on $G_{i}(i=1,2,3)$ should be the same. Almost all papers dealing with measure-perturbed multivalued problems are devoted to the study of only some particular cases of our problem. This is motivated by direct applications of obtained results or there are some problems in the proposed proofs. Moreover, different kind of solutions are considered (mainly due to different applications or proofs). In our opinion, the general case seems to be interesting, because this allows one to indicate some possible extensions in earlier papers.

As can be seen in the next result, by considering similar assumptions as in our existence Theorems 6 or 8 , only for the 'worst' part $\mu_{n s}$, the remaining assumptions can be relaxed. 
In order to do this, we need to recall the definition of a solution for this particular case ( $c f$. [54] or [55]). Let us consider the following problem:

$$
d x(t) \in G_{1}(t, x(t)) d \mu_{a c}(t)+G_{2}(t, x(t)) d \mu_{n s}(t)+G_{3}(t, x(t)) d \mu_{p a}(t), \quad x(0)=x_{0} .
$$

Definition 16 A solution of the problem (8) is a function $x:[0,1] \rightarrow \mathbb{R}^{d}$ such that $x=$ $x_{a c}+x_{n s}+x_{p a}$, where

1. $x_{a c}(t)=x_{0}+\int_{0}^{t} g_{a c}(s) d \mu_{a c}(s)$ for $t \in[0,1]$ and for some $\mu_{a c}$-integrable function $g_{a c}:[0,1] \rightarrow \mathbb{R}^{d}$ and $g_{a c}(t) \in G_{1}\left(t, x\left(t^{-}\right)\right) \mu_{a c}$-a.e.,

2. $\quad x_{n s}(t)=\int_{0}^{t} g_{n s}(s) d \mu_{n s}(s)$ for some $\mu_{n s}$-integrable function $g_{n s}:[0,1] \rightarrow \mathbb{R}^{d}$ and $g_{n s}(t) \in G_{2}\left(t, x\left(t^{-}\right)\right) \mu_{n s}$-a.e.,

3. $\quad x_{p a}(t)=\int_{0}^{t} g_{p a}(s) d \mu_{p a}(s)$ for some $\mu_{p a}$-integrable function $g_{p a}:[0,1] \rightarrow \mathbb{R}^{d}$ and $g_{p a}(t) \in \widetilde{G}_{3}\left(t, x\left(t^{-}\right) ; \mu_{p a}(t)\right) \mu_{p a}$-a.e.

Note that in earlier papers some of the above 'parts' of the solutions are not existing and then Definition 4 is treated as a considered solution (see the corollaries below). In general: the convergence of an integral is expected instead of the approximation property by a sequence of appropriate measures ( $c f$. Definition 14). As claimed in [16] for singlevalued problems (selections) we have

$$
x(t)=x_{0}+\int_{0}^{t} g_{a c}(s) F^{\prime}(s) d s+\int_{0}^{t} g_{n s}(s) d \mu_{n s}(s)+\sum_{t_{k} \leq t, t_{k} \in A} S\left(t_{k}, x\left(t_{k}-\right), \mu\left(\left\{t_{k}\right\}\right)\right),
$$

where $A$ denote a set of atoms for $\mu$ and all the above integrals should be finite (convergent).

The theorem given below allows us to distinguish between different assumptions on every part of the measure $\mu$.

Theorem 17 Assume that:

1. $G_{1}:[0,1] \times \mathbb{R}^{d} \rightarrow \mathcal{P}_{c c}\left(\mathbb{R}^{d}\right)$ is such that $G_{1}(\cdot, \cdot)$ is product Borel measurable, $G_{1}(t, \cdot)$ is usc for every $t \in[0,1]$, there exist a positive function $M_{1} \in L^{1}\left([0,1], \mu_{a c}\right)$ and a constant $N_{1}>0$ such that $G(t, y) \subset\left[M_{1}(t)+N_{1}\|y\|\right]$ for all $t \in[0,1]$ and $y \in \mathbb{R}^{d}$,

2. $G_{2}:[0,1] \times \mathbb{R}^{d} \rightarrow \mathcal{P}_{c c}\left(\mathbb{R}^{d}\right)$ is such that $G_{2}(\cdot, \cdot)$ is product Borel measurable, $G_{2}(t, \cdot)$ is usc for every $t \in[0,1]$, there exists a positive function $M_{2} \in L^{1}\left([0,1], \mu_{n s}\right)$ such that $d(0, G(t, y)) \leq M_{2}(t)$ for all $t \in[0,1]$ and $y \in \mathbb{R}^{d}$,

3. $G_{3}$ is such that $S:[0,1] \times \mathbb{R}^{d} \times[0,1] \rightarrow \mathcal{P}_{c c}\left(\mathbb{R}^{d}\right)$ has compact values and is bounded by $M_{3}$. Moreover the set-valued map $S(t, \cdot, \alpha): \mathbb{R}^{d} \rightarrow \mathbb{R}^{d}$ is usc, uniformly in $t, \alpha \in[0,1]$.

Then there exists at least one solution for the measure-driven differential problem (8).

The proof of the above theorem runs like the proof of our main theorem with an appropriate change of the definition of a sequence $\left(x_{n}\right)$, so we leave a detailed proof to the reader.

The proposed approach via measure-driven inclusions seems to be interesting, because it allows one to unify separately investigated cases for different multivalued problems. Let us recall that in the cases listed below solutions are treated in the sense of Definition 4 . 
This means that there is no general method for an approximation of such solutions (like in our Definition 14) and from this point of view our results are more applicable.

Corollary $18\left[45\right.$, p.98] Let $Q \subset \mathbb{R} \times \mathbb{R}^{d}$ be an open subset containing $\left(0, x_{0}\right)$. Let $F$ be an upper semicontinuous map from $Q$ into the nonempty closed convex subsets of $\mathbb{R}^{d}$. We assume that $(t, x) \rightarrow m(F(t, x))$ is locally compact. Then there exist $T>0$ and an absolutely continuous function $x(\cdot)$ defined on $[0, T], a$ (slow) solution to the differential inclusion $x^{\prime}(t) \in F(t, x(t)), x(0)=x_{0}$.

Here the minimal selection $m(F(t, x))$ of a multifunction $F$ is locally compact in the sense that for each point $(t, x)$ one can find a neighborhood which is mapped into a compact set (see [45, p.97]).

Corollary 19 [9, Corollary 4.2] Let $G_{1}$ and $G_{3}$ have closed convex values, $G_{1}(\cdot, x)$ is measurable, $G_{1}(\cdot, \cdot)$ is product Borel measurable, there exist an integrable function $M$ and a constant $N>0$ such that $G_{1}(t, x) \subset M(t)(1+\|x\|) B$ and $G_{3}(t, x) \subset N(1+\|x\|) B$ for all $x, y \in \mathbb{R}^{n}$. Assume that there exist an integrable function $k$ and a constant $c$ such that

$$
G_{1}(t, x) \subset G_{1}(t, y)+k(t)\|x-y\| B \quad \text { for all } x, y \in \mathbb{R}^{n},
$$

and that $G_{3}$ is Lipschitz:

$$
G_{3}(t, x) \subset G_{3}(t, y)+c\|x-y\| B \quad \text { for all } x, y \in \mathbb{R}^{n} .
$$

Then for any positive Borel measure having the Lebesgue decomposition $\mu=\mu_{a c}+\mu_{p a}$ (i.e. $\left.\mu_{n s} \equiv 0\right)$ and arbitrary $x_{0} \in \mathbb{R}^{n}$ there exists a solution for (8).

For the case of discrete measures let us recall the following result formulated in [56] in the language of dynamic inclusions i.e. inclusions on time scales (cf. also [57]). Here we consider the case $\mathbb{T}=\mathbb{Z}$ and then we have a difference inclusion.

Corollary 20 Let $G_{3}: \mathbb{N} \times \mathbb{R} \rightarrow \mathbb{R}$ have compact convex values, $G_{3}(\cdot, x)$ be measurable, $G_{3}(n, \cdot)$ be usc; and for every $r>0$ assume that there exists an integrable function $M_{r}$ such that $\left\|G_{3}(n, x)\right\| \leq M_{r}(n)$ whenever $\|x\| \leq r$ for all $x \in C(\mathbb{N}, \mathbb{R})$. Then there exists at least one solution for the difference inclusion

$$
y(n+1)-y(n) \in G_{3}(n, y), \quad n \in \mathbb{N} .
$$

Notice that difference inclusions are also investigated without the context of time scales. A similar result to the one presented above can be found in [58, Theorem 2.1] in a direct form of difference inclusions. In [59] difference inclusions are used to solve some problems in optimal control theory. In particular, it was proved that any solution for autonomous differential inclusions can be approximated by a sequence of solutions for difference inclusions (the multifunction $G$ is assumed to be bounded and Lipschitz). A brief discussion of other motivations for difference inclusions can be found in [60] (see also the references therein). We refer to [61] for a full survey of difference methods for differential inclusions. 
Also, the problem for difference inclusions was investigated in the case of impulsive differential inclusions ([62], for instance) and in this setting our approach seems to be natural and does not require separate studies.

Moreover, some results presented for the case of so-called $q$-difference inclusions could be reformulated in our language, but till now they are only particular cases of Corollary 20 .

Competing interests

The authors declare that they have no competing interests.

Authors' contributions

All authors contributed equally and significantly in writing this paper. All authors read and approved the final manuscript.

\section{Author details}

${ }^{1}$ Faculty of Mathematics and Computer Science, A. Mickiewicz University, Umultowska 87, Poznań, 61-614, Poland.

${ }^{2}$ Faculty of Electrical Engineering and Computer Science, 'Stefan cel Mare' University of Suceava, Universitatii 13, Suceava,

Romania.

\section{Acknowledgements}

The authors are deeply indebted to the anonymous reviewers for their valuable suggestions. This work was supported by a grant of the Romanian National Authority for Scientific Research, CNCS-UEFISCDI, project number PN-II-RU-TE-2012-3-0336.

\section{Received: 17 October 2013 Accepted: 20 January 2014 Published: 04 Feb 2014}

\section{References}

1. Aubin, J-P: Impulsive Differential Inclusions and Hybrid Systems: A Viability Approach. Lecture Notes, Univ. Paris (2002)

2. Aubin, J-P, Lygeros, J, Quincampoix, M, Sastry, S, Seube, N: Impulse differential inclusions: a viability approach to hybrid systems. IEEE Trans. Autom. Control 47, 2-20 (2002)

3. Lygeros, J, Quincampoix, M, Rzeżuchowski, T: Impulse differential inclusions driven by discrete measures. In: Hybrid Systems: Computation and Control. Lecture Notes in Computer Science, vol. 4416, pp. 385-398 (2007)

4. Aubin, J-P: Impulse and Hybrid Control Systems: A Viability Approach. A Mini-Course. University of California Press, Berkeley (2002)

5. Moreau, JJ: Bounded variation in time. In: Moreau, JJ, Panagiotopoulos, PD, Strang, G (eds.) Topics in Nonsmooth Mechanics, pp. 1-74. Birkhäuser, Basel (1988)

6. Code, WJ, Loewen, PD: Optimal control of non-convex measure-driven differential inclusions. Set-Valued Var. Anal. $19,203-235(2011)$

7. Goebel, R, Teel, AR: Solutions to hybrid inclusions via set and graphical convergence with stability theory applications. Automatica 42, 573-587 (2006)

8. Sesekin, AN, Fetisova, YV: Functional differential equations in the space of functions of bounded variations. Proc. Steklov Inst. Math. 2, suppl., 258-265 (2010)

9. Silva, GN, Vinter, RB: Measure driven differential inclusions. J. Math. Anal. Appl. 202, 727-746 (1996)

10. Ahmed, NU: Measure solutions impulsive evolution differential inclusions and optimal control. Nonlinear Anal. 47 13-23 (2001)

11. Pereira, FL, Silva, GN: Necessary conditions of optimality for vector-valued impulsive control problems. Syst. Control Lett. 40, 205-215 (2000)

12. Pereira, FL, Silva, GN, Oliveira, V: Invariance for impulsive control systems. Autom. Remote Control 69, 788-800 (2008)

13. Goncharova, E, Staritsyn, M: Optimization of measure-driven hybrid systems. J. Optim. Theory Appl. 153, 139-156 (2012)

14. Filippova, TF: Set-valued solutions to impulsive differential inclusions. Math. Comput. Model. Dyn. Syst. 11, 149-158 (2005)

15. Leine, Rl, van de Wouw, N: Uniform convergence of monotone measure differential inclusions: with application to the control of mechanical systems with unilateral constraints. Int. J. Bifurc. Chaos 18, 1435-1457 (2008)

16. Sesekin, AN, Zavalishchin, ST: Dynamic Impulse Systems. Kluwer Academic, Dordrecht (1997)

17. Kronig, R, Penney, W: Quantum mechanics in crystal lattices. Proc. R. Soc. Lond. 130, 499-513 (1931)

18. Atkinson, FV: Discrete and Continuous Boundary Problems. Academic Press, New York (1964)

19. Sharma, RR: An abstract measure differential equation. Proc. Am. Math. Soc. 32, 503-510 (1972)

20. Shendge, GR, Joshi, SR: Abstract measure differential inequalities and applications. Acta Math. Hung. 41, 53-59 (1983)

21. Dhage, BC, Bellale, SS: Existence theorems for perturbed abstract measure differential equations. Nonlinear Anal. 71 , 319-328 (2009)

22. Schwabik, Š, Tvrdý, M, Vejvoda, O: Differential and Integral Equations. Boundary Problems and Adjoints. Reidel, Dordrecht (1979)

23. Wyderka, Z: Linear Differential Equations with Measures as Coefficients and Control Theory. Wyd. Uniw. Ślaskiego, Katowice (1994)

24. Wyderka, Z: Linear differential equations with measures as coefficients and the control theory. Čas. Pěst. Mat. 114 13-27 (1989)

25. Federson, M, Mesquita, JG, Slavik, A: Measure functional differential equations and functional dynamic equations on time scales. J. Differ. Equ. 252, 3816-3847 (2012) 
26. Wan, $X$, Sun, J: Existence of solutions for perturbed abstract measure functional differential equations. Adv. Differ. Equ. 2011, 67 (2011)

27. Trrdý, M: Differential and Integral Equations in the Space of Regulated Functions. Habil. thesis, Praha (2001)

28. Çamlibel, MK, Heemels, WPMH, van der Schaft, AJ, Schumacher, JM: Solution concepts for hybrid dynamical systems. IFAC (2002)

29. Heemels, WPMH, Camlibel, MK, van der Schaft, AJ, Schumacher, JM: On the existence and uniqueness of solution trajectories to hybrid dynamical systems. In: Nonlinear and Hybrid Control in Automotive Applications, pp. 391-422 (2002)

30. Bogachev, VI: Measure Theory. Springer, Berlin (2007)

31. Johnson, RA: Atomic and nonatomic measures. Proc. Am. Math. Soc. 25, 650-655 (1970)

32. Alberti, G, Mantegazza, C: A note on the theory of SBV functions. Boll. Unione Mat. Ital., B 11, 375-382 (1997)

33. Aliprantis, CD, Border, KC: Infinite Dimensional Analysis. Springer, Berlin (2006)

34. Bruckner, AM, Bruckner, JB, Thomson, BS: Real Analysis. Prentice Hall, New York (1997)

35. Taylor, SJ: Introduction to Measure and Integration. Cambridge University Press, Cambridge (1966)

36. Fremlin, DH: Measure Theory, vol. 2. Torres Fremlin, Colchester (2003)

37. Persson, J: Fundamental theorems for linear measure differential equations. Math. Scand. 62, 19-43 (1988)

38. Persson, J: Regularization of nonlinear measure differential equations. Matematiche 44,113-130 (1989)

39. Aubin, J-P, Frankowska, H: Set-Valued Analysis. Birkhäuser, Boston (1990)

40. Zygmunt, W: On superpositionally measurable semi-Carathéodory multifunctions. Comment. Math. Univ. Carol. 33 73-77 (1992)

41. Wagner, DH: Survey of measurable selection theorems: an update. In: Measure Theory, Oberwolfach 1979 (Proc Conf., Oberwolfach, 1979). Lecture Notes in Math., vol. 794, pp. 176-219. Springer, Berlin (1980)

42. Castaing, C, Valadier, M: Convex Analysis and Measurable Multifunctions. Lecture Notes in Math., vol. 580. Springer, Berlin (1977)

43. Carter, M, van Brunt, B: The Lebesgue-Stieltjes Integral - A Practical Introduction. Springer, Berlin (2000)

44. Diestel, J, Ruess, WM, Schachermayer, W: Weak compactness in $L^{1}(\mu, X)$. Proc. Am. Math. Soc. 118, 447-453 (1993)

45. Aubin, J-P, Cellina, A: Differential Inclusions. Springer, Berlin (1984)

46. Miller, B, Rubinovitch, EY: Impulsive Control in Continuous and Discrete-Continuous Systems. Kluwer Academic, Dordrecht (2003)

47. Seifert, C: Gordon type theorem for measure perturbation. Electron. J. Differ. Equ. 2011, 111 (2011)

48. Constantin, A: Global existence of solutions for perturbed differential equations. Ann. Mat. Pura Appl. (4) CLXVIII, 237-299 (1995)

49. Cichoń, M: Differential inclusions and abstract control problems. Bull. Aust. Math. Soc. 53, 109-122 (1996)

50. Or, Y, Teel, AR: Zeno stability of the set-valued bouncing ball. IEEE Trans. Autom. Control 56, 447-452 (2011)

51. Dal Maso, G, Rampazzo, F: On systems of ordinary differential equations with measures as controls. Differ. Integral Equ. 4, 739-765 (1991)

52. Schechter, E: A survey of local existence theories for abstract nonlinear initial value problems. In: Nonlinear Semigroups, Partial Differential Equations and Attractors. Lecture Notes in Math., vol. 1394, pp. 136-184. Springer, Berlin (1989)

53. Miller, B: Representation of robust and non-robust solutions of nonlinear discrete-continuous systems. In: Hybrid and Real-Time Systems. Lecture Notes in Computer Science, vol. 1201, pp. 228-239 (1997)

54. Sesekin, AN: On sets of discontinuous solutions of nonlinear differential equations. Izv. Vyš̌. Učebn. Zaved., Mat. 6 83-89 (1994) (in Russian)

55. Code, WJ: Measure-Driven Impulsive Systems Stabilization, Optimal Control and Applications. PhD thesis, Vancouver (2009)

56. Atici, FM, Biles, DC: First order dynamic inclusions on time scales. J. Math. Anal. Appl. 292, 222-237 (2004)

57. Frigon, M, Gilbert, H: Systems of first order inclusions on time scales. Topol. Methods Nonlinear Anal. 37, 147-163 (2011)

58. Agarwal, RP, O'Regan, D, Lakshmikantham, V: Discrete second order inclusions. J. Differ. Equ. Appl. 9, 879-885 (2003)

59. Smirnov, GV: Discrete approximations and optimal solutions of differential inclusions. Cybern. Syst. Anal. 27, 101-107 (1991)

60. Kloeden, PE, Marín-Rubio, P: Weak pullback attractors of non-autonomous difference inclusions. J. Differ. Equ. Appl. 9, 489-502 (2003)

61. Dontchev, A, Lempio, F: Difference methods for differential inclusions: a survey. SIAM Rev. 34, 263-294 (1992)

62. Baier, R, Donchev, T: Discrete approximation of impulsive differential inclusions. Numer. Funct. Anal. Optim. 31, 653-678 (2010)

10.1186/1687-1847-2014-56

Cite this article as: Cichoń and Satco: Measure differential inclusions - between continuous and discrete. Advances in Difference Equations 2014, 2014:56 